\title{
Shifted Tietz-Wei oscillator for simulating the atomic interaction in diatomic molecules
}

\author{
Babatunde J. Falaye $^{1} \cdot$ Sameer M. Ikhdair ${ }^{2,4} \cdot$ Majid Hamzavi $^{3}$
}

Received: 23 December 2014/ Accepted: 6 April 2015/Published online: 9 May 2015

(C) The Author(s) 2015. This article is published with open access at Springerlink.com

\begin{abstract}
The shifted Tietz-Wei (sTW) oscillator is as good as traditional Morse potential in simulating the atomic interaction in diatomic molecules. By using the Pekeris-type approximation, to deal with the centrifugal term, we obtain the bound-state solutions of the radial Schrödinger equation with this typical molecular model via the exact quantization rule (EQR). The energy spectrum for a set of diatomic molecules $\left(\mathrm{NO}\left(a^{4} \Pi_{i}\right), \operatorname{NO}\left(B^{2} \Pi_{r}\right)\right.$, $\mathrm{NO}\left(L^{\prime 2} \phi\right), \quad \mathrm{NO}\left(b^{4} \Sigma^{-}\right), \quad \operatorname{ICl}\left(X^{1} \Sigma_{g}^{+}\right), \quad \operatorname{ICl}\left(A^{3} \Pi_{1}\right) \quad$ and $\operatorname{ICl}\left(A^{13} \Pi_{2}\right)$ for arbitrary values of $n$ and $\ell$ quantum numbers are obtained. For the sake of completeness, we study the corresponding wavefunctions using the formula method.
\end{abstract}

Keywords Exact quantization rule - Formula method . Shifted Tietz-Wei potential

Babatunde J. Falaye

fbjames11@physicist.net; babatunde.falaye@fulafia.edu.ng

Sameer M. Ikhdair

sameer.ikhdair@najah.edu; sikhdair@gmail.com

Majid Hamzavi

majid.hamzavi@gmail.com

1 Applied Theoretical Physics Division, Department of Physics, Federal University Lafia, P. M. B. 146, Lafia, Nigeria

2 Department of Physics, Faculty of Science, An-Najah National University, New campus, P. O. Box 7, Nablus, West Bank, Palestine

3 Department of Physics, University of Zanjan, Zanjan, Iran

4 Department of Electrical Engineering, Near East University, Nicosia, Northern Cyprus, Mersin 10, Turkey

\section{Introduction}

By employing the dissociation energy and the equilibrium bond length for a diatomic molecule as explicit parameters, Jia et al. [1] generated improved expressions for some wellknown potentials including Rosen-Morse, ManningRosen, Tietz and Frost-Musulin potential energy functions. These authors found that the well-known Tietz potential function is conventionally defined in terms of five parameters but it actually has only four independent parameters. Furthermore, the Wei [2] and Tietz potential functions [3] are exactly same solvable empirical functions.

Wang et al. [4] also generated improved expressions for two versions of the Schiöberg potential energy function which are the Rosen-Morse and Manning-Rosen potential functions. By choosing the experimental values of the dissociation energy, equilibrium bond length and equilibrium harmonic vibrational frequency as inputs, the authors obtained the average deviations of the energies calculated with the potential model from the experimental data for five diatomic molecules, and find that no one of six three-parameter empirical potential energy functions is superior to the other potentials in fitting experimental data for all molecules examined.

All these efforts were made in an attempt to find a most suitable molecular potential in its description of diatomic molecules. Following Refs. [1, 4], we suggest STW as a modification for the TW [2-8]. This potential can be written as

$$
V(r)=V_{\mathrm{e}}\left[\frac{2\left(c_{\mathrm{h}}-1\right) e^{-b_{h}\left(r-r_{\mathrm{e}}\right)}-\left(c_{\mathrm{h}}^{2}-1\right) e^{-2 b_{h}\left(r-r_{\mathrm{e}}\right)}}{\left(1-c_{\mathrm{h}} e^{-b_{\mathrm{h}}\left(r-r_{\mathrm{e}}\right)}\right)^{2}}\right],
$$

where $b_{\mathrm{h}}=\beta\left(1-c_{\mathrm{h}}\right), r_{\mathrm{e}}$ is the molecular bond length, $\beta$ is the Morse constant, $V_{\mathrm{e}}$ is the potential well depth and $c_{\mathrm{h}}$ is an optimization parameter obtained from ab initio or 
Rydberg-Klein-Rees (RKR) intramolecular potentials. $r$ is the internuclear distance. When the potential constant approaches zero, i.e. $c_{\mathrm{h}} \rightarrow 0$, the sTW potential reduces to the Morse potential [9]. This potential is just the TW potential shifted by dissociation energy $D_{\mathrm{e}}$. The shape of this potential is shown in Fig. 1a for different molecules.

Figure 1b compare between TW diatomic molecular potential, sTW diatomic molecular potential and the Morse potential using the parameters set for $H_{2}\left(X^{1} \Sigma_{g}^{+}\right)$diatomic molecule. As it can be seen from this plot, the shifted Tietz-Wei and the Morse potentials are very close to each other for large values of $r$ in the regions $r \approx r_{\mathrm{e}}$ and $r>r_{\mathrm{e}}$, but they are very different at $r \approx 0$. This implies that the shifted Tietz-Wei potential is as good as traditional Morse potential and better than the Tietz-Wei potential in stimulating the atomic interaction for diatomic molecules.

The scheme of our presentation is as follows. In the next section we give basic ingredient of exact quantization rule and all necessary formulas for our calculations. We solve the radial Schrödinger equation for the sTW and also obtain the rotational-vibrational energy spectrum for some diatomic molecules. Finally, results and conclusions are presented.

\section{A brief review of the exact quantization rule}

Here we give a brief review on the EQR. The details can be found in Refs. [10-24]. In 2005, Ma and Xu [12, 13] by carefully studying one-dimensional Schrödinger equation, have extended results to three-dimensional case by simply making the replacements $x \rightarrow r$ and $V(x) \rightarrow V_{\text {eff }}(r)$ :

$$
\begin{aligned}
& \int_{r_{\mathrm{a}}}^{r_{\mathrm{b}}} k(r) d r=N \pi+\int_{r_{\mathrm{a}}}^{r_{\mathrm{b}}} \phi(r)\left[\frac{\mathrm{d} k(r)}{\mathrm{d} r}\right]\left[\frac{\mathrm{d} \phi(r)}{\mathrm{d} r}\right]^{-1} \mathrm{~d} r, \\
& k(r)=\sqrt{\frac{2 \mu}{\hbar^{2}}\left[E-V_{\mathrm{eff}}(r)\right],}
\end{aligned}
$$

where $r_{A}$ and $r_{B}$ are two turning points determined by $E=V_{\text {eff }}(r)$. The $N=n+1$ is the number of the nodes of $\phi(r)$ in the region $E_{n \ell}=V_{\text {eff }}(r)$ and is larger by one than the number $n$ of the nodes of wave function $\psi(r)$. The first term $N \pi$ is the contribution from the nodes of the logarithmic derivative of wave function, and the second is called the quantum correction.

In this approach, the energy spectrum equation is obtained by solving the two integrals involved in Eq. (2). This quantization rule has been used in many physical systems to obtain the exact solutions of many exactly solvable quantum systems [10-24]. EQR is a very important foundation to proper quantization rule (PQR) [10, 25-27].

\section{The energy spectrum}

To study any quantum physical model characterized by the diatomic molecular potential given by Eq. (1), we need to solve the following Schrödinger equation for spherically symmetric potential in any arbitrary dimensional space:

$$
\begin{aligned}
& \left(\frac{\mathrm{d}^{2}}{\mathrm{~d} r^{2}}+\frac{D-1}{r} \frac{\mathrm{d}}{\mathrm{d} r}-\frac{\ell(\ell+D-2)}{r^{2}}+\frac{2 \mu}{\hbar^{2}}\left(E_{n \ell}-V(r)\right)\right) \\
& \psi_{n, \ell, m}\left(r, \Omega_{D}\right)=0 .
\end{aligned}
$$

Now, by defining the wavefunction $\psi_{n, \ell, m}\left(r, \Omega_{D}\right)$ as $r^{(1-D) / 2} R_{n \ell}(r) Y_{\ell m}(\theta, \phi)$ and taking $V(r)$ as the sTW
Fig. 1 a Shape of sTW diatomic molecular potential for different diatomic molecules. $\mathbf{b}$ Shape of the sTW, TW and Morse oscillator potentials for $H_{2}\left(X^{1} \Sigma_{g}^{+}\right)$diatomic molecule with $c_{\mathrm{h}}=0.170066$, $b_{\mathrm{h}}=1.61890 \AA^{-1}, r_{\mathrm{e}}=$ $0.7416 \AA$ and $V_{\mathrm{e}}=38318 \mathrm{~cm}^{-1}$
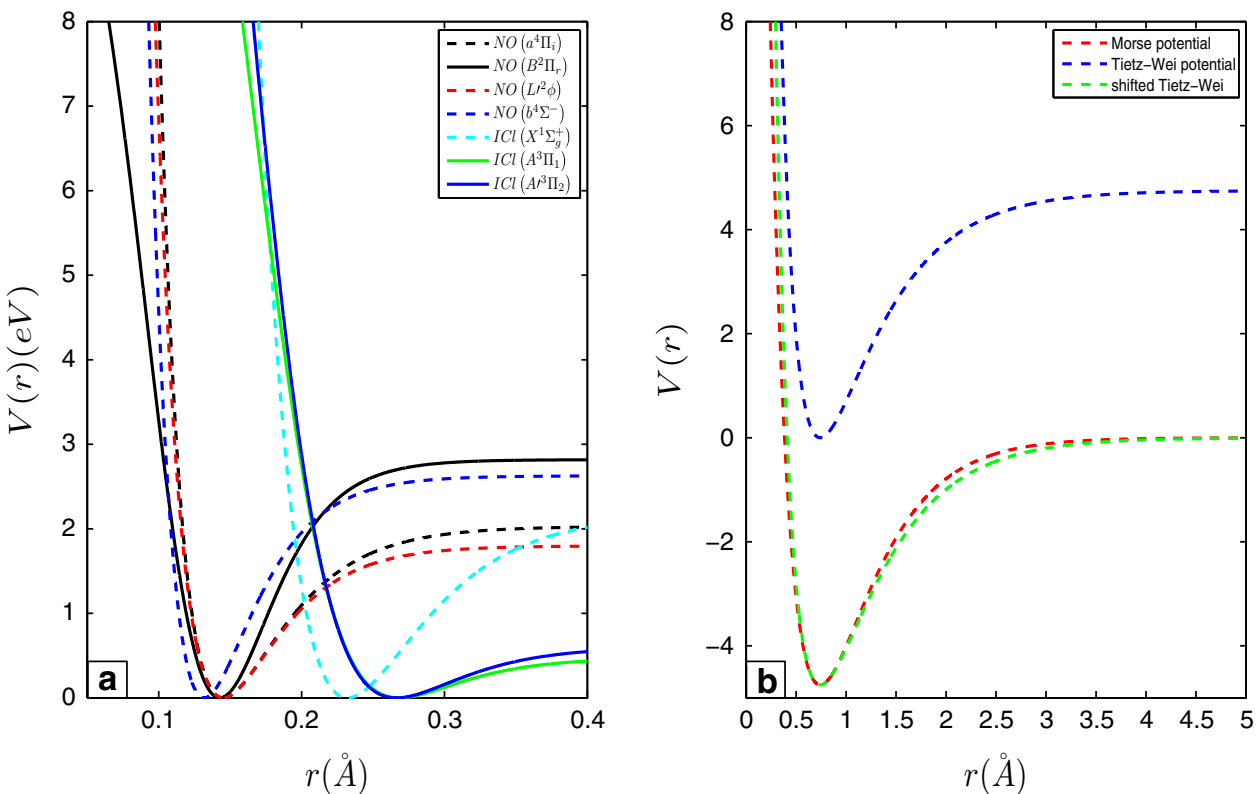
diatomic molecular potential, the radial part of Eq. (3) can be found as

$$
\begin{aligned}
& \frac{\mathrm{d}^{2} R_{n \ell}(r)}{\mathrm{d} r^{2}}+\frac{2 \mu}{\hbar^{2}} \\
& \quad \times\left[E_{n \ell}-V_{\mathrm{e}}\left[\frac{2\left(c_{\mathrm{h}}-1\right) e^{-b_{\mathrm{h}}\left(r-r_{\mathrm{e}}\right)}-\left(c_{\mathrm{h}}^{2}-1\right) e^{-2 b_{h}\left(r-r_{\mathrm{e}}\right)}}{1-c_{\mathrm{h}} e^{-b_{\mathrm{h}}\left(r-r_{\mathrm{e}}\right)}}\right]^{2}\right. \\
& \left.\quad-\frac{\left(\tilde{\eta}^{2}-\frac{1}{4}\right) \hbar^{2}}{2 \mu r^{2}}\right] R_{n \ell}(r)=0
\end{aligned}
$$

where $n, \ell$ and $E_{n \ell}$ denote the principal quantum number, orbital angular momentum number and the bound state energy eigenvalues of the system under consideration (i.e., $\left.E_{n \ell}<0\right)$, respectively. The parameter $\tilde{\eta}=\ell+\frac{1}{2}(D-2)$ which is a linear combination of the spatial dimensions $D$ and the angular momentum quantum number $\ell$. It is well known that for $\ell=0$, problem in the form (4) is exactly solvable. But for $\ell \neq 0$, it is not due to the centrifugal barrier. Therefore, in order to solve the above equation for $\ell \neq 0$ states, Hamzavi et al. [6] found that the following formula is a good approximation scheme to deal with the centrifugal barrier:

$\frac{1}{r^{2}} \approx \frac{1}{r_{\mathrm{e}}^{2}}\left(D_{0}+D_{1} \frac{e^{-b_{\mathrm{h}}\left(r-r_{\mathrm{e}}\right)}}{1-c_{\mathrm{h}} e^{-b_{\mathrm{h}}\left(r-r_{\mathrm{e}}\right)}}+D_{2} \frac{e^{-2 b_{\mathrm{h}}\left(r-r_{\mathrm{e}}\right)}}{\left(1-c_{\mathrm{h}} e^{-b_{\mathrm{h}}\left(r-r_{\mathrm{e}}\right)}\right)^{2}}\right)$,

with

$$
\begin{gathered}
D_{0}=1-\frac{1}{\alpha}\left(1-c_{\mathrm{h}}\right)\left(3+c_{\mathrm{h}}\right)+\frac{3}{\alpha^{2}}\left(1-c_{\mathrm{h}}\right)^{2}, \\
\lim _{c_{\mathrm{h}} \rightarrow 0} D_{0}=1-\frac{3}{\alpha}+\frac{3}{\alpha^{2}} \\
D_{1}=\frac{2}{\alpha}\left(1-c_{\mathrm{h}}\right)^{2}\left(2+c_{\mathrm{h}}\right)-\frac{6}{\alpha^{2}}\left(1-c_{\mathrm{h}}\right)^{3}, \\
\lim _{c_{\mathrm{h}} \rightarrow 0} D_{1}=\frac{4}{\alpha}-\frac{6}{\alpha^{2}} \\
D_{2}=-\frac{1}{\alpha}\left(1-c_{\mathrm{h}}\right)^{3}\left(1+c_{\mathrm{h}}\right)+\frac{3}{\alpha^{2}}\left(1-c_{\mathrm{h}}\right)^{4}, \\
\lim _{c_{\mathrm{h}} \rightarrow 0} D_{2}=-\frac{1}{\alpha}+\frac{3}{\alpha^{2}},
\end{gathered}
$$

Constant $\alpha=b_{\mathrm{h}} r_{\mathrm{e}}$ has been introduced for mathematical simplicity. Now, by inserting this approximation into Eq. (4) and then introducing a new transformation of the form $r \rightarrow \zeta=\frac{r-r_{\mathrm{e}}}{r_{\mathrm{e}}}$ through the mapping function $\zeta=f(r)$ with $r$ in the domain $[0, \infty)$ or $\zeta$ in the domain $[-1, \infty]$, we obtain the following second order differential equation: $\frac{1}{r_{\mathrm{e}}^{2}} \frac{\mathrm{d}^{2} R_{n \ell}(\zeta)}{\mathrm{d} \zeta^{2}}+\frac{2 \mu}{h^{2}}\left[E_{n \ell}-V_{\mathrm{eff}}(\zeta)\right] R_{n \ell}(\zeta)=0, \quad$ with

$$
\begin{aligned}
& V_{\text {eff }}(\zeta)=\left[\mathcal{A}+\frac{\mathcal{B}}{e^{\alpha \zeta}-c_{\mathrm{h}}}+\frac{\frac{\ell(\ell+1) D_{2}}{r_{\mathrm{e}}^{2}}+\frac{2 \mu V_{\mathrm{e}}}{h^{2}}\left(c_{\mathrm{h}}-1\right)^{2}}{\left(e^{\alpha \zeta}-c_{\mathrm{h}}\right)}+\mathcal{F}\left(e^{\alpha \zeta}-c_{\mathrm{h}}\right)^{2}\right], \\
& \mathcal{A}=\left(\frac{\tilde{\eta}^{2}-\frac{1}{4}}{r_{\mathrm{e}}^{2}}\right) \frac{D_{0} h^{2}}{2 \mu}, \mathcal{B}=\left(\frac{\tilde{\eta}^{2}-\frac{1}{4}}{r_{\mathrm{e}}^{2}}\right) \frac{D_{1}-h^{2}}{2 \mu}+2 V_{\mathrm{e}}\left(c_{\mathrm{h}}-1\right), \\
& \mathcal{F}=\left(\frac{\tilde{\eta}^{2}-\frac{1}{4}}{r_{\mathrm{e}}^{2}}\right) \frac{D_{2}-h^{2}}{2 \mu}+V_{\mathrm{e}}\left(c_{\mathrm{h}}-1\right)^{2} .
\end{aligned}
$$

If we define $\tilde{\varsigma}=\frac{1}{e^{\alpha_{\zeta}}-c_{\mathrm{h}}}$, then we can obtain the two turning points $\tilde{\varsigma}_{a}$ and $\tilde{\varsigma}_{b}$ as well as their sum and product properties by solving $V_{\text {eff }}(\zeta)-E_{n \ell}=0$ or $V_{\text {eff }}(\tilde{\varsigma})-E_{n \ell}=0$ as:

$$
\begin{aligned}
& \tilde{\varsigma}_{a}=-\frac{\mathcal{B}}{2 \mathcal{F}}-\frac{1}{2 \mathcal{F}} \sqrt{\mathcal{B}^{2}-4 \mathcal{F}\left(\mathcal{A}-E_{n \ell}\right)}, \quad \text { and } \\
& \tilde{\varsigma}_{b}=\frac{\mathcal{B}}{2 \mathcal{F}}+\frac{1}{2 \mathcal{F}} \sqrt{\mathcal{B}^{2}-4 \mathcal{F}\left(\mathcal{A}-E_{n \ell}\right)} \\
& \tilde{\varsigma}_{a}+\tilde{\varsigma}_{b}=-\frac{\mathcal{B}}{\mathcal{F}}, \quad \tilde{\varsigma}_{a} \tilde{\varsigma}_{b}=\frac{\mathcal{A}-E_{n \ell}}{\mathcal{F}} \quad \text { and } \\
& k(y)=\sqrt{\frac{2 \mu \mathcal{F}}{\hbar^{2}}}\left[-\left(\tilde{\varsigma}-\tilde{\varsigma}_{a}\right)\left(\tilde{\varsigma}-\tilde{\varsigma}_{b}\right)\right]^{1 / 2}
\end{aligned}
$$

Now, we can write the non-linear Riccati equation for the ground state is as

$$
-\alpha \frac{\tilde{\varsigma}(1+\tilde{\varsigma})}{r_{\mathrm{e}}} \phi_{0}^{\prime}(\tilde{\varsigma})+\phi_{0}^{2}(\tilde{\varsigma})+\frac{2 \mu}{\hbar^{2}}\left[E_{0 \ell}-V_{e f f}(\tilde{\varsigma})\right]=0 .
$$

Since the logarithmic derivative $\phi_{0}(\tilde{\varsigma})$ for the ground state has one zero and no pole, it has to take the linear form in $\tilde{\varsigma}$. Thus, we assume the following solution for the ground states

$\phi_{0}(\tilde{\varsigma})=A+B \tilde{\varsigma}$.

By putting Eqs. (10) into (9), we can solve the non-linear Riccati equation. After proper comparison, it is straightforward to obtain the ground state energy and values of $A$ and $B$ as

$E_{0 \ell}=\mathcal{A}-\frac{\hbar^{2} A^{2}}{2 \mu} \quad$ with $\quad A=\frac{\mu}{\hbar^{2}} \frac{\mathcal{B}-\mathcal{F} / c_{\mathrm{h}}^{2}}{B}+\frac{B}{2} \quad$ and

$B=\frac{a c_{\mathrm{h}}}{2 r_{\mathrm{e}}}+\frac{a c_{\mathrm{h}}}{2 r_{\mathrm{e}}} \sqrt{1+\frac{8 \mu \mathcal{F} r_{\mathrm{e}}^{2}}{\alpha^{2} \hbar^{2} c_{\mathrm{h}}^{2}}}$

Since we now have all basic ingredient required to perform our calculations, thus, we proceed to calculating integrals (2) 


$$
\begin{aligned}
& \int_{r_{a}}^{r_{b}} k(r) \mathrm{d} r=-\frac{r_{\mathrm{e}}}{\alpha} \int_{\tilde{\varsigma}_{a}}^{\tilde{\varsigma}_{b}} \frac{k(\tilde{\varsigma})}{a \tilde{\varsigma}(1+\tilde{\varsigma})} d \tilde{\varsigma}=-\frac{r_{\mathrm{e}}}{\alpha} \int_{\tilde{\varsigma}_{0 a}}^{\tilde{\varsigma}_{0 b}} \\
& \times \sqrt{\frac{2 \mu \mathcal{F}}{h^{2}}} \frac{\left[-\left(\tilde{\varsigma}-\tilde{\varsigma}_{a}\right)\left(\tilde{\zeta}-\tilde{\varsigma}_{b}\right)\right]^{1 / 2}}{\tilde{\varsigma}\left(1+\tilde{\varsigma} c_{\mathrm{h}}\right)} \mathrm{d} \tilde{\varsigma} \\
& =-\frac{\pi r_{\mathrm{e}}}{\alpha} \sqrt{\frac{2 \mu \mathcal{F}}{h^{2}}} \\
& \times\left[\frac{\sqrt{\left(1+\tilde{\varsigma}_{a} c_{\mathrm{h}}\right)\left(1+\tilde{\varsigma}_{b} c_{\mathrm{h}}\right)}}{c_{\mathrm{h}}}-\frac{1}{c_{\mathrm{h}}}-\sqrt{\tilde{\varsigma}_{a} \tilde{\varsigma}_{b}}\right] \\
& =-\frac{\pi r_{\mathrm{e}}}{\alpha} \sqrt{\frac{2 \mu \mathcal{F}}{h^{2}}} \\
& \times\left[\frac{\sqrt{\left(\mathcal{A}-E_{n \ell}\right) c_{\mathrm{h}}^{2}-\mathcal{B} c_{\mathrm{h}}+\mathcal{F}}}{\mathcal{F} c_{\mathrm{h}}}-\frac{1}{c_{\mathrm{h}}}-\sqrt{\frac{\mathcal{A}-E_{n \ell}}{\mathcal{F}}}\right],
\end{aligned}
$$

where we have used the following standard integral

$$
\begin{aligned}
& \int_{x_{a}}^{x_{b}} \frac{\sqrt{\left(x_{a}-x\right)\left(x-x_{b}\right)}}{x(1+Q x)} d x \\
& \quad=\pi\left[\frac{\sqrt{\left(Q x_{a}+1\right)\left(Q x_{b}+1\right)}}{Q}-\frac{1}{Q}-\sqrt{x_{a} x_{b}}\right] .
\end{aligned}
$$

Furthermore, we can find the integral on the right hand side as

$$
\begin{aligned}
& \int_{r_{0 a}}^{r_{0 a}} \phi(r)\left[\frac{\mathrm{d} k_{0}(r)}{\mathrm{d} r}\right]\left[\frac{\mathrm{d} \phi(r)}{\mathrm{d} r}\right]^{-1} \mathrm{~d} r \\
& =-\frac{r_{\mathrm{e}}}{\alpha} \int_{\tilde{\zeta}_{0 a}}^{\tilde{\varsigma}_{0 b}}\left[\frac{\mathrm{d} k(r)}{\mathrm{d} r}\right]\left[\frac{\mathrm{d} \phi(\tilde{\zeta})}{\mathrm{d} \tilde{\zeta}}\right]^{-1}\left(\frac{A}{B}+\tilde{\zeta}\right) \mathrm{d} \tilde{\zeta} \\
& =\frac{r_{\mathrm{e}}}{2 \alpha} \int_{\tilde{\varsigma}_{0 a}}^{\tilde{\varsigma}_{0 b}} \sqrt{\frac{2 \mu \mathcal{F}}{\hbar^{2}}} \frac{\left[2 \tilde{\zeta}-\left(\tilde{\varsigma}_{a}+\tilde{\varsigma}_{b}\right)\right]\left[\frac{A}{B}+\tilde{\varsigma}\right]}{\tilde{\varsigma}\left(1+\tilde{\zeta} c_{\mathrm{h}}\right) \sqrt{-\left(\tilde{\zeta}-\tilde{\varsigma}_{a}\right)\left(\tilde{\zeta}-\tilde{\varsigma}_{b}\right)}} \mathrm{d} \tilde{\varsigma} \\
& =\frac{r_{\mathrm{e}}}{2 \alpha} \sqrt{\frac{2 \mu \mathcal{F}}{\hbar^{2}}} \int_{\tilde{\varsigma}_{0 a}}^{\tilde{\tau}_{0 b}}\left[\left(\frac{A}{B}-\frac{1}{c_{\mathrm{h}}}\right)\left(1+\frac{\tilde{\varsigma}_{0 a}+\tilde{\varsigma}_{0 b}}{2} c_{\mathrm{h}}\right) \frac{1}{1+\tilde{\varsigma} c_{\mathrm{h}}}\right. \\
& \left.+\frac{1}{c_{\mathrm{h}}} \cdot-\frac{A}{B}\left(\frac{\tilde{\varsigma}_{0 a}+\tilde{\varsigma}_{0 b}}{2}\right) \frac{1}{\tilde{\varsigma}}\right] \times \frac{\mathrm{d} \tilde{\varsigma}}{\sqrt{-\left(\tilde{\varsigma}-\tilde{\varsigma}_{a}\right)\left(\tilde{\varsigma}-\tilde{\varsigma}_{b}\right)}} \\
& =\left[\left(\frac{A}{B}-\frac{1}{c_{\mathrm{h}}}\right)\left(1-\frac{\mathcal{B} c_{\mathrm{h}}}{2 \mathcal{F}}\right) \frac{1}{\sqrt{1-\frac{\mathcal{B} c_{\mathrm{h}}}{\mathcal{F}}+\frac{\mathcal{A}-E_{0 \ell}}{\mathcal{F}} c_{\mathrm{h}}^{2}}}\right. \\
& \left.+\frac{A}{B}\left(\frac{\mathcal{B}}{2 \mathcal{F}}\right) \frac{\sqrt{\mathcal{F}}}{\sqrt{\mathcal{A}-E_{0 \ell}} \mathcal{F}}\right] \frac{\pi r_{\mathrm{e}}}{\alpha} \sqrt{\frac{2 \mu \mathcal{F}}{\hbar^{2}}} \\
& =\frac{\pi r_{\mathrm{e}}}{\alpha} \sqrt{\frac{2 \mu \mathcal{F}}{\hbar^{2}}}\left[\frac{1}{B c_{\mathrm{h}}} \sqrt{\frac{2 \mu \mathcal{F}}{\hbar^{2}}}+\frac{1}{c_{\mathrm{h}}}\right] \text {. }
\end{aligned}
$$

Using the results in Eqs. (12) and (14) together with Eq. (1), we can find the energy eigenvalues equation for the sTW diatomic molecular potential as

$$
\begin{aligned}
E_{n \ell}^{D}= & \frac{h^{2}\left(\tilde{\eta}^{2}-\frac{1}{4}\right) D_{0}}{2 \mu r_{\mathrm{e}}^{2}}-\frac{\alpha^{2} h^{2}}{2 \mu r_{\mathrm{e}}^{2}} \\
& \times\left[\frac{(\delta+n)^{2}+\frac{\left(\tilde{\eta}^{2}-\frac{1}{4}\right)}{\alpha^{2} c_{\mathrm{h}}^{2}}\left(D_{1} c_{\mathrm{h}}-D_{2}\right)+\frac{2 \mu V_{\mathrm{e}} r_{\mathrm{e}}^{2}}{\alpha^{2} h^{2}}\left(1-\frac{1}{c_{\mathrm{h}}^{2}}\right)}{2(\delta+n)}\right]^{2} \\
& \text { with } \delta=\frac{1}{2}+\frac{1}{2} \sqrt{1+\frac{4}{c_{\mathrm{h}}^{2}}\left(\frac{D_{2}\left(\tilde{\eta}^{2}-\frac{1}{4}\right)}{\alpha^{2}}+\frac{2 \mu V_{\mathrm{e}} r_{\mathrm{e}}^{2}}{\alpha^{2} h^{2}}\left(1-c_{\mathrm{h}}\right)^{2}\right) .}
\end{aligned}
$$

In three-dimensional $(D=3)$ space, it can be reduced to the form

$$
\begin{aligned}
E_{n \ell}= & \frac{h^{2} \ell(\ell+1) D_{0}}{2 \mu r_{\mathrm{e}}^{2}}-\frac{\alpha^{2}-h^{2}}{2 \mu r_{\mathrm{e}}^{2}} \\
& \times\left[\frac{(\delta+n)^{2}+\frac{\ell(\ell+1)}{\alpha^{2} c_{\mathrm{h}}^{2}}\left(D_{1} c_{\mathrm{h}}-D_{2}\right)+\frac{2 \mu V_{e} r_{e}^{2}}{\alpha^{2} h^{2}}\left(1-\frac{1}{c_{h}^{2}}\right)}{2(\delta+n)}\right]^{2} \\
& \text { with } \delta=\frac{1}{2}+\frac{1}{2} \sqrt{1+\frac{4}{c_{h}^{2}}\left(\frac{D_{2} \ell(\ell+1)}{\alpha^{2}}+\frac{2 \mu V_{e} r_{e}^{2}}{\alpha^{2} h^{2}}\left(1-c_{h}\right)^{2}\right) .}
\end{aligned}
$$

\section{The Eigenfunctions}

For the sake of completeness, we study the corresponding wavefunctions for this potential. For this purpose we introduce a new transformation of the form $t=e^{-b_{\mathrm{h}}\left(r-r_{\mathrm{e}}\right)} \in$ $\left(e^{\alpha}, 0\right)$ in Eq. (7), which maintained the finiteness of the transformed wave functions on the boundary conditions. Thus, we can find

$$
\begin{aligned}
& \frac{\mathrm{d}^{2} U_{n \ell}(t)}{\mathrm{d} t^{2}}+\frac{1 \mathrm{~d} U_{n \ell}(t)}{t}+\frac{1}{t^{2}\left(1-c_{\mathrm{h}} t\right)^{2}} \\
& \quad \times\left\{\left[\frac{2 \mu r_{\mathrm{e}}^{2} E_{n \ell}}{\hbar^{2} \alpha^{2}}-\frac{\left(\tilde{\eta}^{2}-\frac{1}{4}\right)}{\alpha^{2}} D_{0}\right]\right. \\
& +\left[-2 c_{\mathrm{h}}\left(\frac{2 \mu r_{\mathrm{e}}^{2}\left(E_{n \ell}+V_{\mathrm{e}}\right)}{\alpha^{2} \hbar^{2}}-\frac{\left(\tilde{\eta}^{2}-\frac{1}{4}\right)}{\alpha^{2}} D_{0}\right)\right. \\
& \left.+\frac{4 \mu r_{\mathrm{e}}^{2} V_{\mathrm{e}}}{\hbar^{2} \alpha^{2}}-\frac{\left(\tilde{\eta}^{2}-\frac{1}{4}\right)}{\alpha^{2}} D_{1}\right] t \\
& +\left[c_{\mathrm{h}}^{2}\left(\frac{2 \mu r_{\mathrm{e}}^{2}\left(E_{n \ell}+V_{\mathrm{e}}\right)}{\alpha^{2} \hbar^{2}}-\frac{\left(\tilde{\eta}^{2}-\frac{1}{4}\right)}{\alpha^{2}} D_{0}\right)\right. \\
& \left.\left.+\frac{\left(\tilde{\eta}^{2}-\frac{1}{4}\right)}{\alpha^{2}}\left(D_{1} c_{\mathrm{h}}-D_{2}\right)-\frac{2 \mu r_{\mathrm{e}}^{2} V_{\mathrm{e}}}{\hbar^{2} \alpha^{2}}\right] t^{2}\right\} U_{n \ell}(t)=0 .
\end{aligned}
$$

Following the procedure described in Ref. [28], we can write the solution $U_{n \ell}(t)$ in terms of hypergeometric polynomials and thus the wave function takes the form 
Table 1 Model parameters of the diatomic molecules studied in the present work

\begin{tabular}{llllll}
\hline Molecules (states) & $c_{\mathrm{h}}$ & $\mu / 10^{-23}(\mathrm{~g})$ & $b_{\mathrm{h}}\left(\AA^{-1}\right)$ & $r_{\mathrm{e}}(\AA)$ & $D\left(\mathrm{~cm}^{-1}\right)$ \\
\hline NO $\left(a^{4} \Pi_{i}\right)$ & 0.0082003 & 1.249 & 2.408413 & 1.451 & 16,361 \\
NO $\left(B^{2} \Pi_{r}\right)$ & -0.482743 & 1.249 & 3.42650 & 1.428 & 22,722 \\
NO $\left(L^{\prime 2} \phi\right)$ & -0.073021 & 1.249 & 2.73796 & 1.451 & 14,501 \\
NO $\left(b^{4} \Sigma^{-}\right)$ & -0.085078 & 1.249 & 3.01538 & 1.318 & 21,183 \\
ICl $\left(X^{1} \Sigma_{g}^{+}\right)$ & -0.086212 & 4.55237 & 2.008578 & 2.3209 & 17,557 \\
ICl $\left(A^{3} \Pi_{1}\right)$ & -0.167208 & 4.55237 & 2.542557 & 2.6850 & 3814.7 \\
ICl $\left(A^{\prime 3} \Pi_{2}\right)$ & -0.157361 & 4.55237 & 2.373450 & 2.6650 & 4875 \\
\hline
\end{tabular}

Table 2 The bound states energy eigenvalues $(D=2$ and 3) for set of diatomic molecules for various $n$ and rotational $\ell$ quantum numbers in sDF diatomic molecular potential

\begin{tabular}{|c|c|c|c|c|c|c|c|c|c|}
\hline D & $n$ & $\ell$ & $\mathrm{NO}\left(a^{4} \Pi_{i}\right)$ & $\mathrm{NO}\left(B^{2} \Pi_{r}\right)$ & $\mathrm{NO}\left(L^{\prime 2} \phi\right)$ & NO $\left(b^{4} \Sigma^{-}\right)$ & $\mathrm{ICl}\left(X^{1} \Sigma_{g}^{+}\right)$ & $\mathrm{ICl}\left(A^{3} \Pi_{1}\right)$ & $\mathrm{ICl}\left(A^{\prime 3} \Pi_{2}\right)$ \\
\hline & 0 & 0 & -1.971298585 & -2.88233770 & -1.855428285 & -2.695026855 & -2.200695845 & -0.4861418795 & -0.618432865 \\
\hline & 1 & 0 & -1.859308585 & -3.01386965 & -1.972859085 & -2.834614855 & -2.248819245 & -0.5129225795 & -0.646829015 \\
\hline & & 1 & -1.859148585 & -3.01373250 & -1.972723585 & -2.834451355 & -2.248804845 & -0.5129118495 & -0.646818105 \\
\hline & 2 & 0 & -1.750608585 & -3.14759050 & -2.093631485 & -2.977436555 & -2.297420945 & -0.5403157895 & -0.675774025 \\
\hline & & 1 & -1.750468585 & -3.14745280 & -2.093493685 & -2.977270755 & -2.297406745 & -0.5403049695 & -0.675763045 \\
\hline & & 2 & -1.750098585 & -3.14703970 & -2.093081285 & -2.976773655 & -2.297363645 & -0.5402725095 & -0.675730195 \\
\hline \multirow[t]{15}{*}{2} & 3 & 0 & -1.645178585 & -3.28347662 & -2.217724085 & -3.123472855 & -2.346500045 & -0.5683148895 & -0.705263165 \\
\hline & & 1 & -1.645068585 & -3.28333841 & -2.217584185 & -3.123304955 & -2.346485745 & -0.5683039695 & -0.705252125 \\
\hline & & 2 & -1.644678585 & -3.28292375 & -2.217165185 & -3.122801255 & -2.346442645 & -0.5682712195 & -0.705219005 \\
\hline & & 3 & -1.644058585 & -3.28223274 & -2.216466785 & -3.121961855 & -2.346370345 & -0.5682166495 & -0.705163785 \\
\hline & 4 & 0 & -1.543078585 & -3.42150558 & -2.345115985 & -3.272704955 & -2.396055245 & -0.5969133695 & -0.735291785 \\
\hline & & 1 & -1.542938585 & -3.42136686 & -2.344973985 & -3.272534755 & -2.396040945 & -0.5969023495 & -0.735280655 \\
\hline & & 2 & -1.542598585 & -3.42095075 & -2.344548485 & -3.272024355 & -2.395997445 & -0.5968693195 & -0.735247275 \\
\hline & & 3 & -1.541978585 & -3.42025729 & -2.343839285 & -3.271173455 & -2.395924945 & -0.5968142695 & -0.735191635 \\
\hline & & 4 & -1.541118585 & -3.41928647 & -2.342846285 & -3.269982555 & -2.395823745 & -0.5967371995 & -0.735113745 \\
\hline & 0 & 0 & -1.971278585 & -2.88230356 & -1.855395085 & -2.694986655 & -2.200692145 & -0.4861392095 & -0.618430175 \\
\hline & 1 & 0 & -1.859278585 & -3.01383536 & -1.972825385 & -2.834574055 & -2.248815545 & -0.5129198795 & -0.646826285 \\
\hline & & 1 & -1.859008585 & -3.01356106 & -1.972554585 & -2.834247155 & -2.248787045 & -0.5128984495 & -0.646804535 \\
\hline & 2 & 0 & -1.750568585 & -3.14755607 & -2.093596885 & -2.977395155 & -2.297417445 & -0.5403130995 & -0.675771295 \\
\hline & & 1 & -1.750288585 & -3.14728068 & -2.093321985 & -2.977063955 & -2.297388745 & -0.5402914395 & -0.675749345 \\
\hline & & 2 & -1.749808585 & -3.14672991 & -2.092771985 & -2.976400855 & -2.297331445 & -0.5402481695 & -0.675705525 \\
\hline \multirow[t]{9}{*}{3} & 3 & 0 & -1.645158585 & -3.28344206 & -2.217689085 & -3.123430855 & -2.346496645 & -0.5683121595 & -0.705260395 \\
\hline & & 1 & -1.644908585 & -3.28316563 & -2.217409585 & -3.123095155 & -2.346467645 & -0.5682903295 & -0.705238345 \\
\hline & & 2 & -1.644418585 & -3.28261279 & -2.216850885 & -3.122423255 & -2.346410245 & -0.5682466595 & -0.705194145 \\
\hline & & 3 & -1.643678585 & -3.28178358 & -2.216012785 & -3.121415755 & -2.346323545 & -0.5681811695 & -0.705127915 \\
\hline & 4 & 0 & -1.543028585 & -3.42147091 & -2.345080285 & -3.272662455 & -2.396051945 & -0.5969106095 & -0.735288995 \\
\hline & & 1 & -1.542798585 & -3.42119348 & -2.344796885 & -3.272322355 & -2.396022645 & -0.5968885995 & -0.735266735 \\
\hline & & 2 & -1.542328585 & -3.42063869 & -2.344229385 & -3.271641355 & -2.395964745 & -0.5968445495 & -0.735222225 \\
\hline & & 3 & -1.541588585 & -3.41980654 & -2.343378185 & -3.270620455 & -2.395877945 & -0.5967784695 & -0.735155455 \\
\hline & & 4 & -1.540608585 & -3.41869709 & -2.342243285 & -3.269259655 & -2.395761945 & -0.5966903895 & -0.735066435 \\
\hline
\end{tabular}




$$
\begin{aligned}
& U_{n \ell}(\zeta)=N_{n \ell} e^{-p \alpha \zeta}\left(1-c_{\mathrm{h}} e^{-\alpha \zeta}\right)^{q}{ }_{2} F_{1}(-n, n+2(p+q) ; \\
& \left.2 p+1, c_{\mathrm{h}} e^{-\alpha \zeta \zeta}\right),
\end{aligned}
$$

with

$$
\begin{aligned}
& p=\sqrt{\left[\left(\frac{\tilde{\eta}^{2}-\frac{1}{4}}{\alpha^{2}}\right) D_{0}-\frac{2 \mu r_{\mathrm{e}}^{2}}{\hbar^{2} \alpha^{2}} E_{n \ell}\right]} \text { and } \\
& q=\frac{1}{2}\left\{1+\sqrt{1+\frac{4}{c_{\mathrm{h}}^{2}}\left[\left(\frac{\tilde{\eta}^{2}-\frac{1}{4}}{\alpha^{2}}\right) D_{2}+\frac{2 \mu r_{\mathrm{e}}^{2} V_{\mathrm{e}}}{\hbar^{2} \alpha^{2}}\left(1-c_{\mathrm{h}}\right)^{2}\right]}\right\},
\end{aligned}
$$

where $N_{n \ell}$ is the normalization constant. For a further detail on the calculation of similar potential models solved using formula method, one is advised to refer to other work [28]

\section{Results and conclusions}

In this study, in an attempt to find a more suitable potential that simulate the atomic interaction in diatomic molecules, we suggested sTW diatomic molecular potential as a modification for the TW diatomic molecular potential. The bound state solution of this potential has been found in an arbitrary D-dimension via the improved exact quantization rule.

Further, using the spectroscopic parameters presented in Table 1 which are taken from Ref. [8], we computed rotational-vibrational energy spectrum of some diatomic molecules in 2,3,4,5-dimensions. The results are presented in Tables 2 and 3. In our numerical computations, we have

\begin{tabular}{|c|c|c|c|c|c|c|c|c|c|}
\hline D & $n$ & $\ell$ & $\mathrm{NO}\left(a^{4} \Pi_{i}\right)$ & $\mathrm{NO}\left(B^{2} \Pi_{r}\right)$ & $\mathrm{NO}\left(L^{\prime 2} \phi\right)$ & $\mathrm{NO}\left(b^{4} \Sigma^{-}\right)$ & $\mathrm{ICl}\left(X^{1} \Sigma_{g}^{+}\right)$ & $\mathrm{ICl}\left(A^{3} \Pi_{1}\right)$ & $\mathrm{ICl}\left(A^{\prime 3} \Pi_{2}\right)$ \\
\hline & 0 & 0 & -1.971158585 & -2.88220112 & -1.855295085 & -2.694865755 & -2.200681645 & -0.4861312495 & -0.61842210 \\
\hline & 1 & 0 & -1.859148585 & -3.01373250 & -1.972723585 & -2.834451355 & -2.248804845 & -0.5129118495 & -0.646818105 \\
\hline & & 1 & -1.858778585 & -3.01332103 & -1.972317885 & -2.833961155 & -2.248762245 & -0.5128796695 & -0.646785525 \\
\hline & 2 & 0 & -1.750468585 & -3.14745280 & -2.093493685 & -2.977270755 & -2.297406745 & -0.5403049695 & -0.675763045 \\
\hline & & 1 & -1.750098585 & -3.14703970 & -2.093081285 & -2.976773655 & -2.297363645 & -0.5402725095 & -0.675730195 \\
\hline & & 2 & -1.749438585 & -3.14635128 & -2.092393885 & -2.975945655 & -2.297292045 & -0.5402183995 & -0.675675405 \\
\hline \multirow[t]{15}{*}{3} & 3 & 0 & -1.645068585 & -3.28333841 & -2.217584185 & -3.123304955 & -2.346485745 & -0.5683039695 & -0.705252125 \\
\hline & & 1 & -1.644678585 & -3.28292375 & -2.217165185 & -3.122801255 & -2.346442645 & -0.5682712195 & 5219005 \\
\hline & & 2 & -1.644058585 & -3.28223274 & -2.216466785 & -3.121961855 & -2.346370345 & -0.5682166495 & 163785 \\
\hline & & 3 & -1.643188585 & -3.28126535 & -2.215488785 & -3.120786155 & -2.346269545 & -0.5681402395 & -0.705086495 \\
\hline & 4 & 0 & -1.542938585 & -3.42136686 & -2.344973985 & -3.272534755 & -2.396040945 & -0.5969023495 & -0.735280655 \\
\hline & & 1 & -1.542598585 & -3.42095075 & -2.344548485 & -3.272024355 & -2.395997445 & -0.5968693195 & 247275 \\
\hline & & 2 & -1.541978585 & -3.42025729 & -2.343839285 & -3.271173455 & -2.395924945 & -0.5968142695 & -0.735191635 \\
\hline & & 3 & -1.541118585 & -3.41928647 & -2.342846285 & -3.269982555 & -2.395823745 & -0.5967371995 & -0.735113745 \\
\hline & & 4 & -1.540048585 & -3.41803836 & -2.341569785 & -3.268451355 & -2.395693345 & -0.5966380895 & -0.735 \\
\hline & 0 & 0 & -1.970998585 & -2.88203041 & -1.855128785 & -2.694664455 & -2.200663845 & -0.4861179795 & -0.618408615 \\
\hline & 1 & 0 & -1.859008585 & -3.01356106 & -1.972554585 & -2.834247155 & -2.248787045 & -0.5128984495 & -0.64680453 \\
\hline & & 1 & -1.858508585 & -3.01301246 & -1.972013385 & -2.833593555 & -2.248729945 & -0.5128555495 & -0.64676106 \\
\hline & 2 & 0 & -1.750288585 & -3.14728068 & -2.093321985 & -2.977063955 & -2.297388745 & -0.5402914395 & -0.675749345 \\
\hline & & 1 & -1.749808585 & -3.14672991 & -2.092771985 & -2.976400855 & -2.297331445 & -0.5402481695 & -0.675705525 \\
\hline & & 2 & -1.749048585 & -3.14590380 & -2.091946885 & -2.975407055 & -2.297245545 & & -0.675639815 \\
\hline \multirow[t]{9}{*}{4} & 3 & 0 & -1.644908585 & -3.28316563 & -2.217409585 & -3.123095155 & -2.346467645 & -0.5682903295 & -0.705238345 \\
\hline & & 1 & -1.644418585 & -3.28261279 & -2.216850885 & & -2.346410245 & & -0.705194145 \\
\hline & & 2 & -1.643678585 & -3.28178358 & -2.216012785 & & -2.346323545 & & -0.705127915 \\
\hline & & 3 & -1.642668585 & -3.28067802 & -2.214895385 & -3.120072855 & -2.346208245 & -0.5680938395 & -0.705039565 \\
\hline & 4 & 0 & -1.542798585 & -3.42119348 & -2.344796885 & -3.272322355 & -2.396022645 & -0.5968885995 & -0.735266735 \\
\hline & & 1 & -1.542328585 & -3.42063869 & -2.344229385 & -3.271641355 & -2.395964745 & -0.5968445495 & -0.735222225 \\
\hline & & 2 & -1.541588585 & -3.41980654 & -2.343378185 & -3.270620455 & -2.395877945 & -0.5967784695 & -0.735155455 \\
\hline & & 3 & -1.540608585 & -3.41869709 & -2.342243285 & -3.269259655 & -2.395761945 & -0.5966903895 & -0.735066435 \\
\hline & & 4 & -1.539388585 & -3.41731030 & -2.340824985 & -3.267557955 & -2.395617345 & -0.5965802895 & -0.734955135 \\
\hline
\end{tabular}
used the following conversions: $1 \mathrm{amu}=931.494028$

Table 3 The bound states energy eigenvalues ( $D=4$ and 5) for set of diatomic molecules for various $n$ and rotational $\ell$ quantum numbers in sDF diatomic molecular potential 
$\mathrm{MeV} / \mathrm{c}^{2}, \quad 1 \mathrm{~cm}^{-1}=1.239841875 \times 10^{-4} \quad \mathrm{eV}, \quad$ and $\hbar c=1973.29 \mathrm{eV} \AA$.

From Eq. (15), it can be seen that two interdimensional states are degenerate whenever $(n, \ell, D) \rightarrow(n, \ell \pm 1, D \mp$ 2) $\Rightarrow E_{n, \ell}^{D}=E_{n, \ell \pm 1}^{(D \mp 2)}$. Thus, a knowledge of $E_{n, \ell}^{D}$ for $\mathrm{D}=2$ to 5 provides the information necessary to find $E_{n, \ell}^{D}$ for other higher dimensions. For example, $E_{0,4}^{(2)}=E_{0,3}^{(4)}=E_{0,2}^{(6)}=E_{0,1}^{(8)}$. This is the same transformational invariance described for bound states of free atoms and molecules [29-31] and demonstrates the existence of interdimensional degeneracies among states of the confined Hulthén potential.

The advantage of the approach employed in this study is that it gives the eigenvalues through the calculation of two integral given by Eq. (2) and solving the resulting algebraic equation. Firstly, we can easily obtain the quantum correction by only considering the solution of the ground state of the quantum system since it is independent of the number of nodes of the wave function for exactly solvable quantum system. The general expressions obtained for the energy eigenvalues and wave functions can be easily reduced to the $3 \mathrm{D}$ space $(\mathrm{D}=3)$ and for s-wave (i.e. $\ell=0$ state). The EQR produce as good results as the $\mathrm{PQR}$, however the procedure followed using $\mathrm{PQR}$ is more shorter and quick.

Acknowledgments We thank the kind referees for the positive enlightening comments and suggestions, which have greatly helped us in making improvements to this paper. In addition, BJF acknowledges eJDS (ICTP) and Prof. K. J. Oyewumi, for his encouragement.

Open Access This article is distributed under the terms of the Creative Commons Attribution 4.0 International License (http:// creativecommons.org/licenses/by/4.0/), which permits unrestricted use, distribution, and reproduction in any medium, provided you give appropriate credit to the original author(s) and the source, provide a link to the Creative Commons license, and indicate if changes were made.

\section{References}

1. Jia, C.S., Diao, Y.F., Liu, X.J., Wang, P.Q., Liu, J.Y., Zhang, G.D.: Equivalence of the Wei potential model and Tietz potential model for diatomic molecules. J. Chem. Phys. 137, 014101 (2012)

2. Hua, W.: Four-parameter exactly solvable potential for diatomic molecules. Phys. Rev. A. 42, 2524 (1990)

3. Tietz, T.: Potential energy function for diatomic molecules. J. Chem. Phys. 38, 3036 (1963)

4. Wang, P.Q., Zhang, L.H., Jia, C.S., Liu, J.Y.: Equivalence of the three empirical potential energy models for diatomic molecules. J. Mol. Spect. 274, 5 (2012)

5. Gordillo-Vázquez, F.J., Kunc, J.A.: Radial probability distributions of atoms in diatomic molecules represented by the rotating Morse and Tietz-Hua oscillators. J. Mol. Struct. (THEOCHEM) 425, 263 (2012)

6. Falaye, B.J., Oyewumi, K.J., Ikhdair, S.M., Hamzavi, M.: Eigensolution techniques, their applications and Fisher's information entropy of the Tietz-Wei diatomic molecular model. Phys. Scr. 89, 115204 (2014)

7. Sun, G.H, Dong, S.H.: Relativistic treatment of spinless particles subject to a Tietz-Wei oscillator. Commun. Theor. Phys. 58, 195 (2012)

8. Gordillo-Vázquez, F.J., Kunc, J.A.: Statistical-mechanical calculations of thermal properties of diatomic gases. J. Appl. Phys. 84, 4693 (1998)

9. Ikhdair, S.M.: Effective Schrödinger equation with general ordering ambiguity position-dependent mass Morse potential. Mol. Phys. 110, 1415 (2012)

10. Serrano, F.A., Gu, X.Y., Dong, S.H.: Qiang-Dong proper quantization rule and its applications to exactly solvable quantum systems. J. Math. Phys. 51, 082103 (2010)

11. Dong, S.H., Cruz-Irisson, M.: Energy spectrum for a modified Rosen-Morse potential solved by proper quantization rule and its thermodynamic properties. J. Math. Chem. 50, 881 (2012)

12. Ma, Z.Q., Xu, B.W.: Quantum correction in exact quantization rules. Europhys. Lett. 69, 685 (2005)

13. Ma, Z.Q., Xu, B.W.: Exact quantization rules for bound states of the Schrödinger equation. Int. J. Mod. Phys. E. 14, 599 (2005)

14. Yang, C.N.: Monopoles in quantum field theory. In: Craigie, N.S., Goddard, P., Nahm, W. (eds.) Proceedings of the Monopole Meeting, Trieste, Italy, p. 237. World Scientific, Singapore (1982)

15. Gu, X.Y., Dong, S.H.: Energy spectrum of the Manning-Rosen potential including centrifugal term solved by exact and proper quantization rules. J. Math. Chem. 49, 2053 (2011)

16. Kasri, Y., Chetouani, L.: Application of the exact quantization rule for some noncentral separable potentials. Can. J. Phys. 86, 1083 (2008)

17. Ikhdair, S.M., Sever, R.: Exact quantization rule to the Kratzertype potentials: an application to the diatomic molecules. J. Math. Chem. 45, 1137 (2009)

18. Qiang, W.C., Zhou, R.S., Gao, Y.: Application of the exact quantization rule to the relativistic solution of the rotational Morse potential with pseudospin symmetry. J. Phys. A: Math. Theor. 40, 1677 (2007)

19. Dong, S.H., Morales, D., Garcia-Ravelo, J.: Exact quantization rule and its applications to physical potentials. Int. J. Mod. Phys. E 16, 189 (2007)

20. Qiang, W.C., Dong, S.H.: Arbitrary 1-state solutions of the rotating Morse potential through the exact quantization rule method. Phys. Lett. A 363, 169 (2007)

21. Gu, X.Y., Dong, S.H., Ma, Z.Q.: Energy spectra for modified Rosen-Morse potential solved by the exact quantization rule. J. Phys. A: Math. Theor. 42, 035303 (2009)

22. Ikhdair, S.M., Abu-Hasna, J.: Quantization rule solution to the Hulthén potential in arbitrary dimension with a new approximate scheme for the centrifugal term. Phys. Scr. 83, 025002 (2011)

23. Falaye, B.J., Ikhdair, S.M., Hamzavi, M.: Energy states of some diatomic molecules: the exact quantization rule approach. ZFN A. 70, 85 (2015)

24. Ikhdair, S.M., Sever, R.: Any 1-state solutions of the Woodssaxon potential in arbitrary dimensions within the new improved quantization rule. Int. J. Mod. Phys. A 25, 3941 (2010)

25. Qiang, W.C., Dong, S.H.: Proper quantization rule. EPL 89, 10003 (2010)

26. Serrano, F.A., Cruz-Irisson, M., Dong, S.H.: Proper quantization rule as a good candidate to semiclassical quantization rules. Ann. Phys. (Berlin) 523, 771 (2011)

27. Falaye, B.J., Ikhdair, S.M., Hamzavi, M.: Spectroscopic study of some diatomic molecules via the proper quantization rule. J. Math. Chem. (2015). doi:10.1007/s10910-015-0491-9

28. Falaye, B.J., Ikhdair, S.M., Hamzavi, M.: Formula method for bound state problems. Few-Body Syst. 56, 63 (2015) 
29. Herrick, D.R.: Degeneracies in energy levels of quantum systems of variable dimensionality. J. Math. Phys. 16, 281 (1975)

30. Herrick, D.R., Stillinger, F.H.: Variable dimensionality in atoms and its effect on the ground state of the helium isoelectronic sequence. Phys. Rev. A 11, 42 (1975)
31. Frantz, D.D., Herschbach, D.R.: Interdimensional degeneracy and symmetry breaking in $D$-dimensional $\mathrm{H}_{2}^{+}$. The J. Chem. Phys. 92 (11), 6668 (1990) 